\title{
PCI, polarisation and exchange effects in (e,2e) collisions
}

\author{
C.T. WHELAN, R.J. ALLAN* and H.R.J. WALTERS**
}

Department of Applied Mathematics and Theoretical Physics, University of Cambridge, Silver St, CB3 9EW, U.K.

* SERC Daresbury Laboraton, Warrington WA4 4AD, U.K.

${ }^{* *}$ Department of Applied Mathematics and Theoretical Physics, The Queen's University of Belfast, $B T 7$ INN, Northern Ireland

\begin{abstract}
The role of post-collisional, polarisation and exchange effects in energy sharing (e,2e) collisions is considered. Methods of including such effects are assessed. Results are presented for the ionisation of hydrogen and helium gases in coplanar geometry.

Results are also presented for the ionisation of helium, argon and xenon in perpendicular plane geometry.
\end{abstract}

1.Introduction The ionisation of atoms at energies above threshold in energy sharing geometries has proven to be a delicate process. The triple differential cross section (TDCS) is remarkably sensitive to subtle kinematical and dynamical effects; three-body influences are felt in the initial and final channels and an accurate description of the Physics presents a substantial challenge to theory. In this paper we consider how, within the framework of a relatively simple approximation we may assess the influence of polarisation, exchange and the post-collisional forces between the particles in the final state. Our approach is essentially explorative, our object is to identify kinematical arrangements where the cross section will be sensitive to these effects, thus allowing both theory and experiment to focus on these regions in the pursuit of a complete description. It should be emphasised from the outset that there may be interferences e.g. when the influence of the Coulomb three-body interactions are strong in the initial channel they may also be strong in the final one, and the inclusion of one without the other may give only a poor shape to the TDCS.

In the description of energy-sharing geometries the Distorted Wave Born Approximation (DWBA) has proven itself to be remarkably useful for a wide range of energies and geometries (Whelan et al. [1]). Indeed a Póst-Collision Interaction (PCI)-modified version has given good results both as to the form and absolute size of the experimental cross section for the ionisation of $\mathrm{H}$ and $\mathrm{He}$ just a few $\mathrm{eV}$ above threshold , $[2,3,4]$.

There are however measurements on He at 26.6,30,40 and $50 \mathrm{eV}$ incident energies, [5], in coplanar symmetric geometry where the approximation simply does not work. In a previous paper,[1], we showed that the general form of the experiment could be reproduced within the context of a modified DWBA approximation with a Gamow factor used to represent the post-collisional interactions. This approximation, which we will denote WAWZ, is crude and should be regarded only as a first step towards a better theory. It does have the advantage, however, that PCI, polarisation and exchange can be switched on and off at will. By strict analogy with helium we can define potentials for hydrogen and we can predict a narrow energy range where similar effects will be strong.

One curious fact about the ionisation of He at low energies is that DWBA fails in coplanar symmetric geometry at energies where it continues to work quite well in the perpendicular plane. 
A simple intuitive model has been proposed, [7], to explain how the two final state electrons could escape in the perpendicular plane.

2.Ionisation of Helium in the perpendicular plane

In a recent set of experiments, [6], measurements were made of the TDCS for the ionisation process

$$
e^{-}+H e\left(1 s^{2}\right) \rightarrow e^{-}+e^{-}+H e^{+}(1 s),
$$

in energy sharing perpendicular plane geometry. In this geometry the two outgoing electrons, momenta $\mathbf{k}_{s}, \mathbf{k}_{f}$, in the final state lie in a plane perpendicular to the incident momentum $\mathbf{k}_{0}$ i.e

$$
\left\|\mathbf{k}_{f}\right\|=\left\|\mathbf{k}_{s}\right\|, \quad \mathbf{k}_{f} \cdot \mathbf{k}_{0}=\mathbf{k}_{s} \cdot \mathbf{k}_{0}=0 \quad \& \quad \mathbf{k}_{f} \cdot \mathbf{k}_{s}=\mathbf{k}_{f}{ }^{2} \cos \phi .
$$

The experimental results are presented as a function of the angle $\phi$ between the two detected electrons. It should be noted that while $\phi$ is quoted in the range $0 \leq \phi \leq 360^{\circ}$, the ranges $0 \leq \phi \leq 180^{\circ}, 180^{\circ} \leq \phi \leq 360^{\circ}$, are physically the same.

For a free collision between an incident and a stationary electron resulting in two outgoing electrons we must have $\mathbf{k}_{0}, \mathbf{k}_{f}, \mathbf{k}_{s}$ in the same plane with $\phi=90^{\circ}$. The first question a theoretician must ask, therefore, is how do the final electrons get into a plane perpendicular to the incident direction?

Zhang et al,[7], Whelan et al,[1], have considered this problem and noted that the atomic electron is neither free nor stationary but rather in a bound state with a momentum distribution. These authors envisaged ionisation into the perpendicular plane as proceeding through one of two simple mechanisms.

Single Scattering

The incident electron collides with an electron of the distribution whose momentum $\mathbf{k}$ exactly cancels the motion of the incident electron in the $\mathbf{k}_{0}$ direction ie $\mathbf{k}=\kappa-\mathbf{k}_{0}$ where $\kappa \cdot \mathbf{k}_{0}=0$ then both electrons will emerge in the perpendicular plane with $\mathbf{k}_{f}+\mathbf{k}_{s}=\kappa$. Now for a $\mathrm{He}\left(1 \mathrm{~s}^{2}\right)$ target the momentum distribution is peaked at $\mathbf{k}=\mathbf{0}$ and decreases rapidly as $\mathrm{k}$ increases. Since $k=\sqrt{\kappa^{2}+k_{0}^{2}}$ the most likely value of $\kappa$ is is zero with the result that $\mathbf{k}_{f}=-\mathbf{k}_{s} \Rightarrow \phi=180^{\circ}$.

Double Scattering

The incident electron first elastically scatters off the atom (essentially the nucleus) with $\mathbf{k}_{0}$ changing to $\mathbf{k}_{0}{ }^{\perp}$, where $\left\|\mathbf{k}_{0}{ }^{\perp}\right\|=\left\|\mathbf{k}_{0}\right\|$. Then in a second energy and momentum conserving free collision, with a bound electron of momentum $\mathbf{k}$, ionisation takes place with

$$
\mathbf{k}_{0}{ }^{\perp}+\mathbf{k}=\mathbf{k}_{f}+\mathbf{k}_{s}
$$

As before, the most probable value of $\mathbf{k}$ is 0 and in this case $\mathbf{k}_{0}{ }^{\perp}$ must be perpendicular to $\mathbf{k}_{0}$ and the angle between $\mathbf{k}_{f}$ and $\mathbf{k}_{s}$ is $90^{\circ}$. As $\left\|\mathbf{k}_{0}\right\|$ increases it becomes much more difficult for the the bound electron to ionise via the single scattering mechanism, while the most probable value of the momentum for a double collision remains at $\mathbf{k}=0$. Zhang et al [7] therefore predicted that at sufficiently low energies that single scattering would give the main contribution to the TDCS with a strong peak at $180^{\circ}$, with double scattering becoming more important as the energy increases. This latter mechanism will give rise to a peak at $90^{\circ}$ which should eventually become dominant.

Motivated by this simple intuitive model calculations have been performed in the distorted wave Born approximation (DWBA), [1].

In this approximation the TDCS for a Helium target is given by :

$$
\frac{d^{3} \sigma}{d \Omega_{f} d \Omega_{s} d E}=2(2 \pi)^{4} \frac{k_{f} k_{s}}{k_{0}}\left(|f|^{2}+|g|^{2}-\operatorname{Re}\left(f^{*} g\right)\right)
$$

where

$$
\begin{aligned}
& f=\left\langle\chi^{-}\left(\mathbf{k}_{f}, \mathbf{r}_{f}\right) \chi^{-}\left(\mathbf{k}_{s}, \mathbf{r}_{s}\right)\left|r_{12}^{-1}\right| \chi^{+}\left(\mathbf{k}_{0}, \mathbf{r}_{f}\right) \psi_{1 s}\left(r_{s}\right)>,\right. \\
& g=\left\langle\chi^{-}\left(\mathbf{k}_{f}, \mathbf{r}_{s}\right) \chi^{-}\left(\mathbf{k}_{s}, \mathbf{r}_{f}\right)\left|r_{12}^{-1}\right| \chi^{+}\left(\mathbf{k}_{0}, \mathbf{r}_{f}\right) \psi_{1 s}\left(r_{s}\right)>,\right.
\end{aligned}
$$

$\psi_{1 s}\left(r_{s}\right)$ is the $1 s$ orbital of $\mathrm{He}\left(1 s^{2}\right), \chi^{+}$is the distorted wave for the incident electron generated in the static exchange potential of the atom and ${ }^{*}$ denotes complex conjugation. The distorted waves $\chi^{-}$are generated in the static exchange potential of the ion and then each is orthogonalised to $\psi_{1 s}\left(r_{s}\right)$. The orbital $\psi_{1 s}\left(r_{s}\right)$ is taken from the Hartree- Fock wave functions of Clement and Roetti,[8]. 
$104.6 \mathrm{eV}$
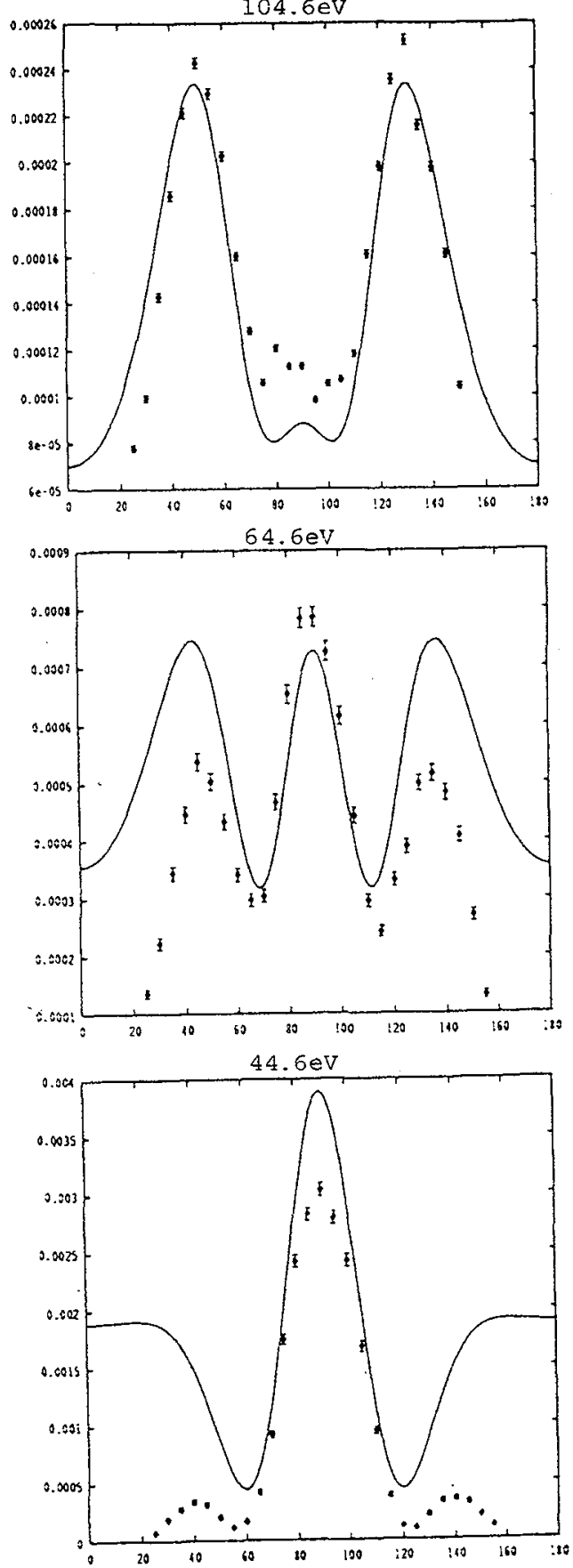

$84.6 \mathrm{eV}$

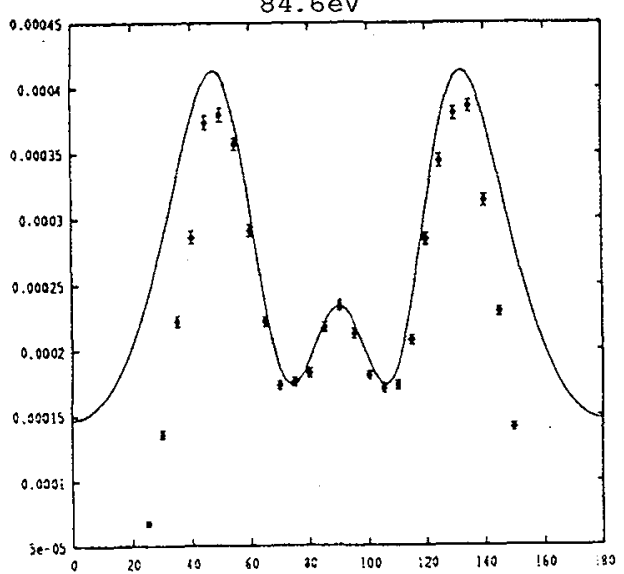

$54.6 \mathrm{eV}$

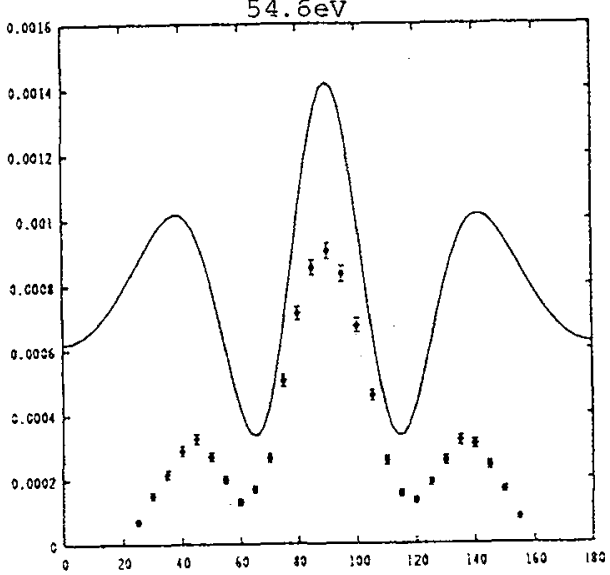

Figure 1

TDCS for the ionisation of $\mathrm{He}$

in energy sharing perpendicular plane geometry for various incident energies. DWBA approximation with spin singlet static exchange, spectator electron has 'atomic'wave function.

Experiment normalised to theory at $104.6 \mathrm{eV}$.

Results are for TDCS against $\phi / 2$

where $\phi$ is the angle between the electrons. 
It is important to note what approximation (3) does and does not not contain. It does not allow for capture of the incident electron into the final state of the $\mathrm{He}^{+}$ion, it takes no account of polarisation nor of the interaction between the two exiting electrons. What approximation (3) does include is the single and double scattering mechanisms discussed above. A number of points should be mentioned concerning the technical implementation of (3): exchange is included only by using the local approximation of Furness and McCarthy, $[9,10]$. Because of the symmetry of the geometry one is obliged to treat both final state active electrons in the same way and forced to deal with a series of ambiguities for example we have a choice between a spin singlet or spin triplet static exchange potential for the outgoing electrons, we have a choice in how we represent the ion potential eg we can assume that the wavefunction of the atomic 'spectator ' electron is the exact $\mathrm{He}^{+}$wavefunction-or to invoke a 'sudden' approximation and use $\mathrm{He}$ atom wavefunction for the spectator electron; ionisation into the perpendicular plane in a delicate process and, particularly at the lower energies, it can be sensitive to these choices.

In Figure 1 we compare the experimental data,[6], with the DWBA calculations of Whelan et al [1] , calculated using a spin static singlet exchange potential and the 1s orbital of the He atom ('sudden approximation') to evaluate the final distorted waves. Although the absolute size of the measurements is not determined experimentally, the relative size at different energies is fixed so that normalisation to theory at only one energy and angle is permitted. We have normalised at $104.6 \mathrm{eV}$ since the theory is expected to be best at higher energies. It is seen from the figure that the pattern of the experimental results is quite well reproduced by the DWBA calculations; further, the predictions of the simple ZWW model would appear to be justified.

\section{Ionisation of $\mathrm{He}$ in coplanar symmetric geometry}

In this geometry the two outgoing electrons have equal energies and their momenta $\mathbf{k}_{f}, \mathbf{k}_{s}$ lie in the same plane as the incident momentum, $\mathbf{k}_{0}$ and make the same angle $\theta$ with the direction of $\mathbf{k}_{0}$. For energies of $100 \mathrm{eV}$ and above there is good agreement between the DWBA calculations and experiment (Whelan et al [1], Pochat et al [11]) both as to the shape and the absolute size of the cross-section. (For full discussion see [1]). (Figure 2a)

The Kaiserslautern group, [5], have extended their coplanar measurements in Helium below $100 \mathrm{eV}$; here there is no agreement with DWBA (calculated from (3)) Figure $2 b, c, d$. The contrast between this picture and that of Figure 1 is striking, DWBA being more successful in the perpendicular plane.

Earlier, we mentioned that our DWBA does not take account of capture of the incident electron into the final ion state, nor of electron-electron repulsion in the final state, nor of polarisation of the target by the incoming electron. All of which might be quite important at the lower energies; not only as effects in themselves but also as they interfere with each other. The effect of the electron-electron repulsion in the perpendicular plane would tend to enhance the peak at $\phi=180^{\circ}$.

In order to test for the significance of polarisation and final state PCI Whelan et al,[1], modified the DWBA calculations as follows: an ad hoc polarisation potential was added in the initial and final states channels i.e

$$
\begin{aligned}
V_{\text {polarisation }} & =-\frac{\alpha}{2 r^{4}} \\
& =-\frac{\alpha}{2 r_{0}^{4}}
\end{aligned}
$$$$
r>r_{0}
$$$$
r \leq r_{0}
$$

where $\alpha$ is the polarisability of neutral $H e\left(1 s^{2}\right)(1.39)$ for the incident channel and the polarisability of $\mathrm{He}^{+}(.28125)$ for the outgoing channels, in both cases they took $r_{0}=.7565$. They then calculated

$$
\frac{d^{3} \sigma^{W A W Z}}{d \Omega_{f} d \Omega_{s} d E}=N_{e e} \frac{d^{3} \sigma^{D W B A}}{d \Omega_{f} d \Omega_{s} d E}
$$

where

$$
N_{e e}=\frac{\gamma}{e^{\gamma}-1}
$$

with

$$
\gamma=\frac{2 \pi}{\left\|\mathbf{k}_{f}-\mathbf{k}_{s}\right\|}
$$

This same $N_{e e}$ factor was introduced by Botero and Macek,[12], in their improved final state Coulomb Born approximation. Its effect is to give the dominant angular behaviour of the TDCS 

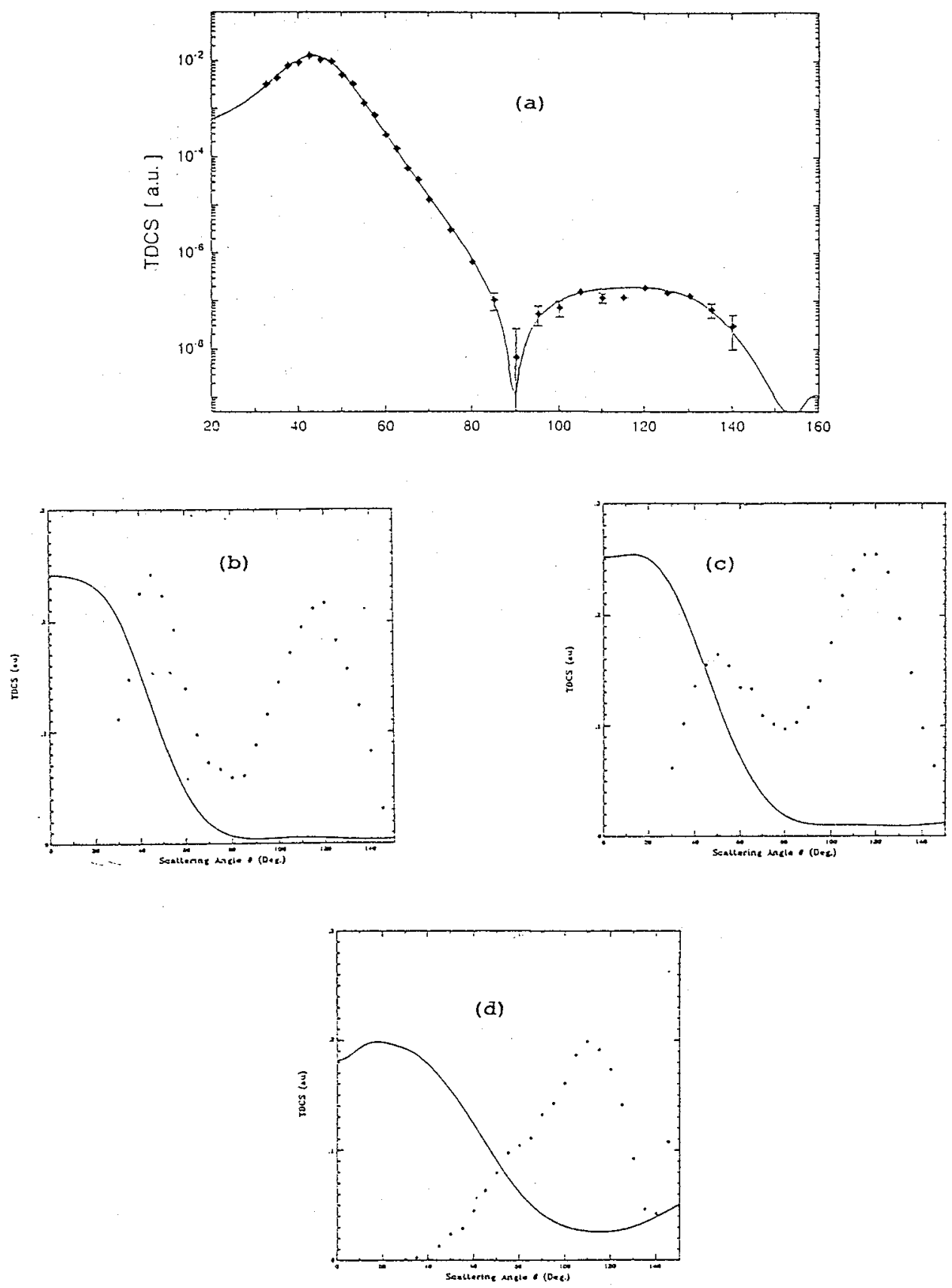

Figure 2

TDCS for the ionisation of He in coplanar symmetric geometry at impact energies of (a) $500 \mathrm{eV}$, (b) $50 \mathrm{eV}$,

(c) $40 \mathrm{eV}$, (d) $30 \mathrm{eV}$. Theory DWBA,[1], Experiment, [5]. 
at low energies due to final state electron-electron interaction. Unfortunately the overall normalisation introduced by $N_{e e}$ is unreliable so we can no longer have confidence in the absolute size of the theoretical TDCS.

We show in Figure 9 the DWBA (singlet and triplet) results in the coplanar case at 50,40,30eV where agreement with the shape of the experimental data is quite good, and at $54.6 \mathrm{eV}$ in both coplanar and perpendicular plane geometries. In the latter case it produces quite a reasonable shape for the central peak but the wings observed in the experiment are now far too big.

We remark that we can not reproduce a good shape for the cross section in coplanar symmetric geometry without using both the $N_{e e}$ term and the polarisation potential in our DWBA calculations. This contrasts with the Coulomb Born calculations of Botero and Macek,[12], which uses only the $N_{e e}$ factor but needs no polarisation potential. We speculate that the use of a bare Coulomb potential ( with a variable charge $Z_{\text {eff }}$ to represent static field screening) in the incident channel employed in their approach is giving the extra attractive interaction which comes from the polarisation potential in our work. It would be very interesting to explore experimentally geometries where either the polarisation/correlation effects or the pci effects might be thought to dominate. For example the $N_{e e}$ factor is purely geometric and could be fixed simply by keeping $\left\|\mathbf{k}_{\mathbf{f}}-\mathbf{k}_{\mathbf{s}}\right\|$ constant.

Very recently Ehrhardt and his collaborators have reported experiments on $\mathrm{He}$,[2], in coplanar geometry in which the two final-state electrons are emitted at $\phi=180$ degrees to each other. Results are presented as a function of the angle $\theta_{f}$ between $\mathbf{k}_{f}$ and the incident direction $\mathbf{k}_{0}$. We have $\theta_{s}=180-\theta_{f}$, and $\left\|\mathbf{k}_{f}\right\|=\left\|\mathbf{k}_{s}\right\|$

Calculations in the DWBA model have been made previously by Pan and Starace, [4], and by Jones et al,[3]. In both the calculations distorted waves used in the final channel were calculated using momentum dependent effective charges $\left(z_{s}, z_{f}\right)$ (see eg Whelan et al [1]) to define the potentials which obeyed the asymptotic conditions

$$
\begin{aligned}
& \lim _{r \rightarrow 0} V_{s, f}(r) \rightarrow \frac{-Z}{r} \\
& \lim _{r \rightarrow \infty} V_{s, f}(r) \rightarrow \frac{-z_{s, f}}{r}
\end{aligned}
$$

where $\mathrm{Z}$ is the nuclear charge.

Pan and Starace used the Peterkop relation to fix the effective charges

$$
\frac{z_{s}}{k_{s}}+\frac{z_{f}}{k_{f}}=\frac{1}{k_{s}}+\frac{1}{k_{f}}-\frac{1}{\left\|\mathbf{k}_{f}-\mathbf{k}_{s}\right\|}
$$

which in this geometry, with $k=k_{s}=k_{f}, \mathbf{k}_{s} \cdot \mathbf{k}_{f}=k^{2} \cos (\pi)$ reduces to

$$
z_{s}=z_{f}=3 / 4
$$

Jones et al use a slightly different prescription which in this geometry yields

$$
z_{s}=z_{f}=1 / 2
$$

In this very particular geometry one would intuitively expect the Peterkop relation, (6), to work well. We might imagine the electrons to exit from the reaction with straight line trajectories. Asymptotically, in a simple picture, one would have

$$
k_{s} / r_{s}=k_{f} / r_{j}=t
$$

where $\mathbf{t}$ is a constant (the time in a classical picture) and Peterkop relation is equivalent to writing

$$
\frac{z_{s}}{k_{s}}+\frac{z_{f}}{k_{f}}=\frac{1}{k_{s}}+\frac{1}{k_{f}}-\frac{1}{\left\|\mathbf{k}_{f}-\mathbf{k}_{s}\right\|}
$$

The treatments of Pan and Starace and Jones and Madison implicitly include PCI effects through the effective charges. The former moreover also included an exact treatment of the exchange interaction. 
45
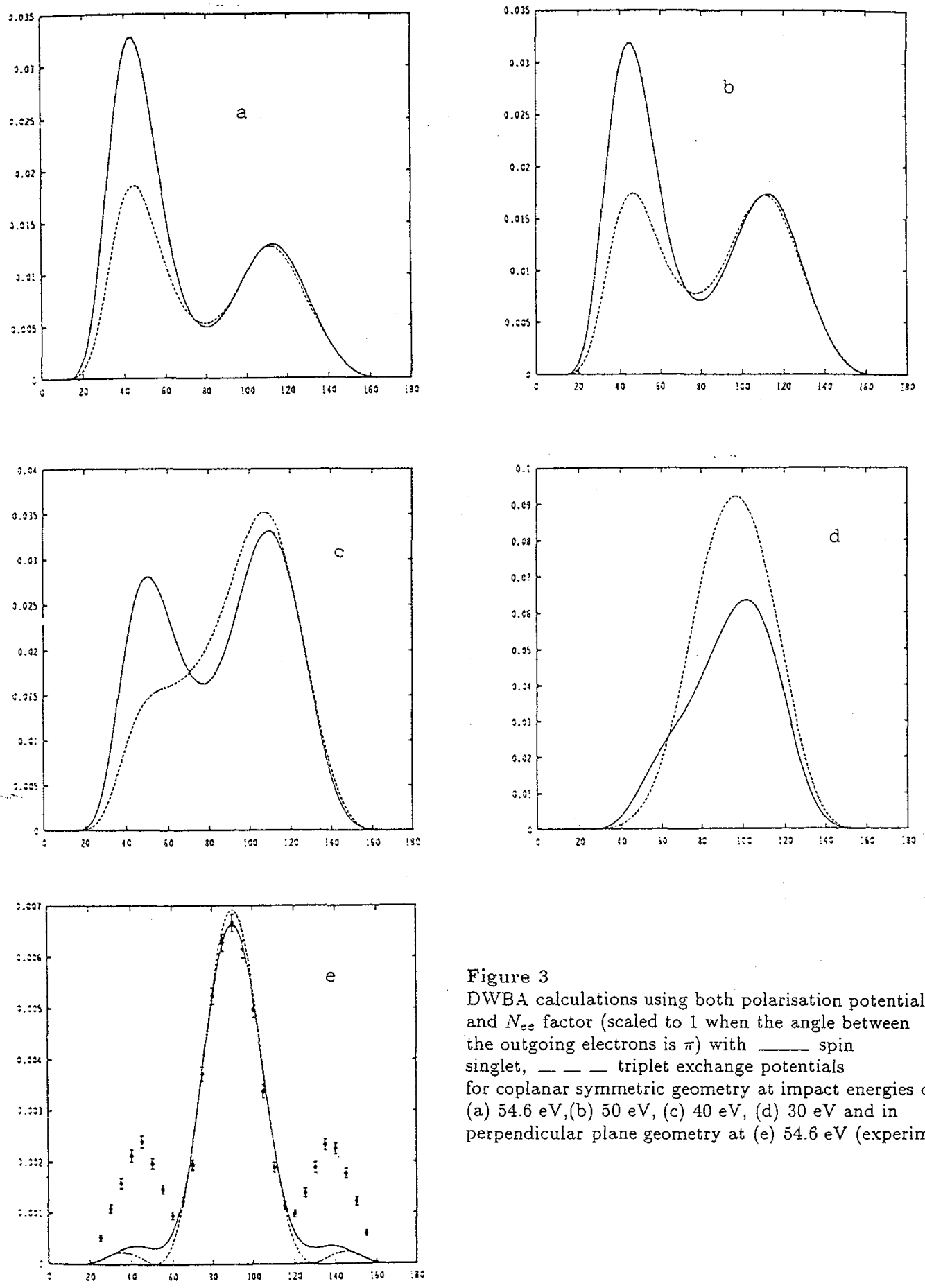

Figure 3

DWBA calculations using both polarisation potential and $N_{e s}$ factor (scaled to 1 when the angle between the outgoing electrons is $\pi$ ) with __ spin singlet, _ - - triplet exchange potentials for coplanar symmetric geometry at impact energies of (a) $54.6 \mathrm{eV}$,(b) $50 \mathrm{eV}$, (c) $40 \mathrm{eV}$, (d) $30 \mathrm{eV}$ and in perpendicular plane geometry at (e) $54.6 \mathrm{eV}$ (experiment[6]). 
We have repeated DWBA calculations using the prescription of Pan and Starace, but using the local static exchange approximation instead of exact exchange. It is found that an "average" local exchange model lies close to the earlier results as shown in Figure 4. We have applied this approximation to the calculation of the TDCS for the ionisation of $\mathrm{He}$ at $50 \mathrm{eV}$ in coplanar symmetric geometry. It was not successful even with the inclusion of polarisation potentials Figure 5. The strength of the polarisation potential used in the WAWZ model for helium was essential chosen to give good accord with experiment at $50 \mathrm{eV}$. We have extended the model to hydrogen where the polarisation potential was defined by analogy with $\mathrm{He}$, ie for $\alpha$ we used the polarisability of $\mathrm{H}$ and we used a cutoff defined by

$$
r_{0}^{H}=r_{0}^{H e} \frac{<r>_{H}}{<r>_{H e}}
$$

where $\langle r\rangle$ denotes the expectation value of $r$. We find a similar double peak structure but at a much lower energy Figure 6 . We strongly urge experiment in this energy range since if the structure predicted by the WAWZ model could be confirmed then it would be an ideal focus for more sophisticated theories. *

4.p-states of Noble Gases in the Perpendicular Plane

In Figure 7 we show the ionisation of $\operatorname{Ar}(3 \mathrm{p})$ in energy sharing perpendicular plane geometry for a range of energies. We see that at low impact energies we have a minimum at $\mathbf{k}_{\mathbf{f}}=-\mathbf{k}_{\mathbf{s}}$.

As the energy of impact is increased a maximum develops.

Secondary peaks develop as the impact energy is increased still further.

These calculations were performed in the DWBA approximation using a sudden approximation for the atomic ion and the singlet form of the static exchange potential.

Similar behaviour has been found for $\mathrm{Xe}(5 \mathrm{p})$ and one result is shown at $94.6 \mathrm{eV}$ incident energy in Figure 8 . The fact that the secondary peak arises from the double-scattering mechanism was confirmed by replacing the distorted wave in the incident channel by a plane wave (dashed curve in the figure). We note that no pci effects are included, were these included the large wings would be reduced to zero at $\mathbf{k}_{\mathbf{f}}=\mathbf{k}_{\mathbf{s}}$. A much fuller discussion of perpendicular plane ionisation will be given elsewhere.

5. Conclusions

3-body effects are important in both the initial and final channels, the rather crude approximation we have introduced suggests this strongly. However a much better theoretical description is needed. Effective charge prescription work very well in the special geometry where $\mathbf{k}_{\mathbf{s}}=-\mathbf{k}_{f}$ but are found lacking when this condition is violated. However if one were primarily interested in studying target wave function effects one might consider their use in this restricted geometry. Results are presented which suggest that some interesting measurements could be obtained on $\mathrm{Ar}$ and $\mathrm{Kr}$ targets in the perpendicular plane. It has been suggested that one should look at the ionisation of $\mathrm{H}(1 \mathrm{~s})$ in coplanar symmetric geometry at impact energies of $20 \mathrm{eV}$ in order to see strong 3 body effects. It has been further proposed that one should consider geometries where $\left\|\mathbf{k}_{f}-\mathbf{k}_{s}\right\|$ is held constant.

These geometries would be suitable not only for studies of polarisation effects but also for $(e, 3 e),(\gamma, 2 e)$ studies whose principal object was to probe target wave function of highly correlated systems. At low impact ejection energies for the two electrons pci will be strong but it is at the lower energies that the cross-section will be greatest. However in the geometries we propose the effect pci should be approximately constant.

\section{Acknowledgments}

This work has been supported by the British German Academic Research Collaboration Programme, the SERC, EEC and by a NATO collaborative research grant (CRG 920101). Much of the computations for this work were carried out on the Intel iPSC/ 860 hypercube at Daresbury Laboratory.

\section{References}

[1]Whelan C.T., Allan R.J., Walters H.R.J. and Zhang X. in "(e,2e) and related processes" C.T.Whelan, H.R.J.Walters, A.Lahmam-Bennani and H.Ehrhardt edited(Kluwer Academic Publishers: 1993)

* We note that during the meeting Dr K. Jung for the University of Kaiserslautern has reported that such experiments have been performed and that the double peak structure of the WAWZ model has been found at the energy predicted 


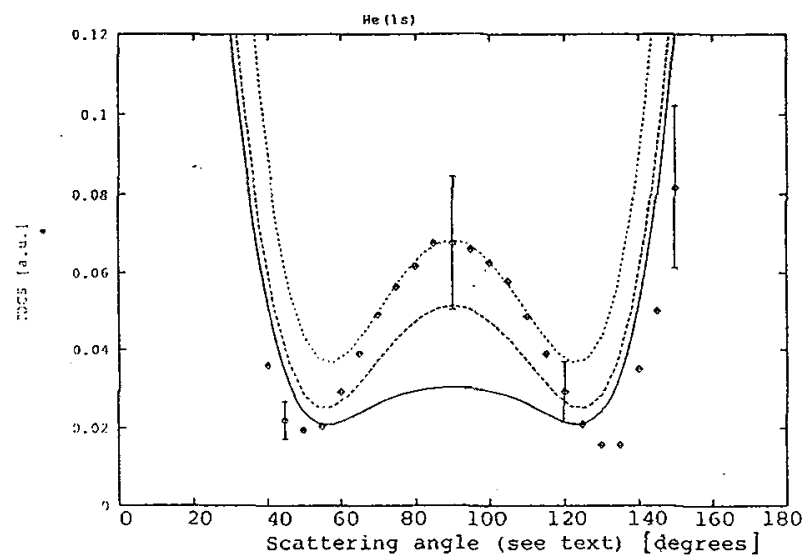

Figiue $4 \mathrm{IIC}$ (Is) coplanar 180 degree geometry results at $4 \mathrm{eV}$ above threshold. Experimental results,[2]. DWBA calculations effective charge proscription with P'eterkop asymptotics long dashed line, singlch potcnital; short dashed linc, average spin potential; dotted tine, triplet potential.

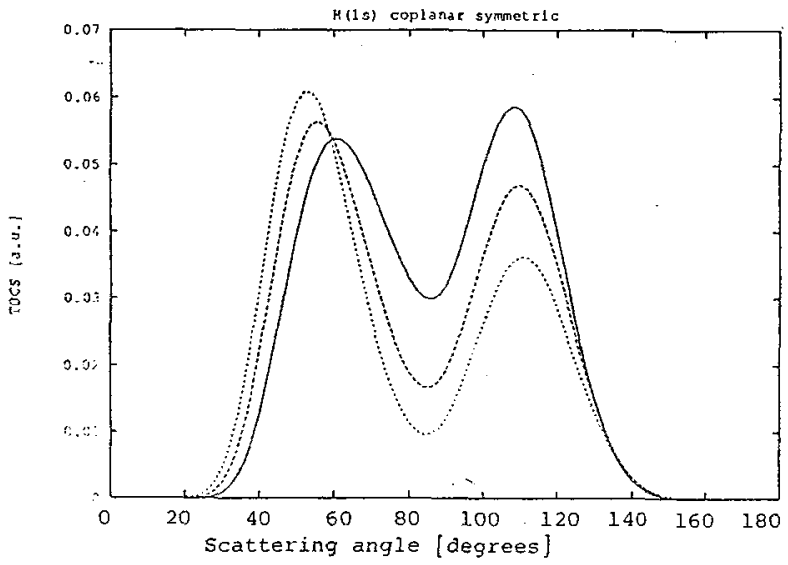

Figure $511(1 \mathrm{~s})$ in coplanar symmetric geometry calculated in the IVAWZ model as desctibed in text with spin singlet exchange potential for impact energies above threshold of $6 \mathrm{eV}$, solid line, $8 \mathrm{eV}$, dashed line, $10 \mathrm{eV}$, short dashed lite.

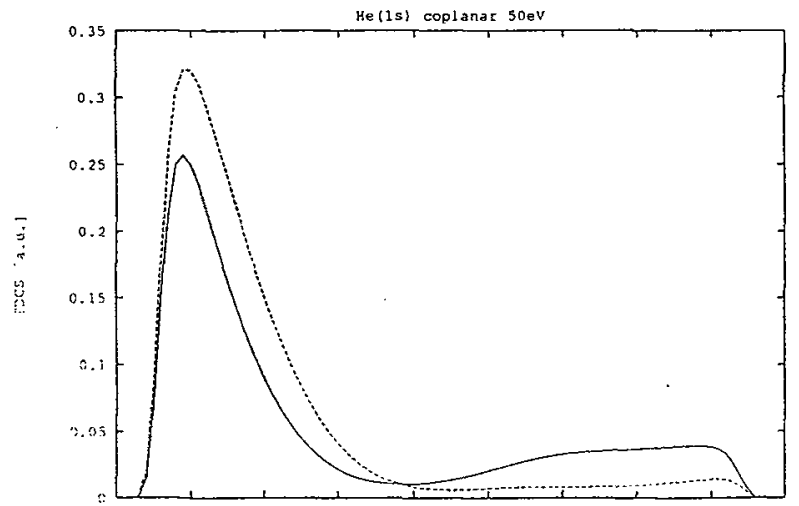

Figure 6 He(ls) ionisation in coplanar symmetric geometry at $50 \mathrm{cV}$ impact energy compared with DWBA, spin avcraged exchange potential, using effective charges with Peterkop asymptotics with, and without, _- polarisation potenlials as described in text. 

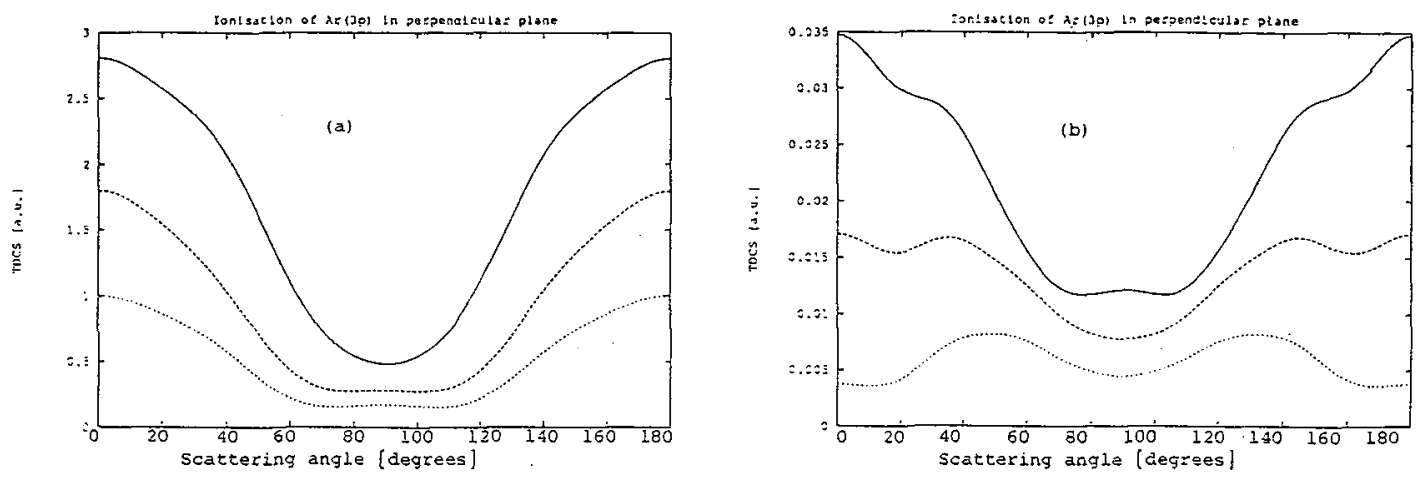

Figure 7 Ionisation of $\operatorname{Ar}(3 p)$ in the perpendicular plane (a) at incident electron energy. (b) at energies $65.7 \mathrm{eV}$,

21. $\mathrm{TeV}$, $25.7 \mathrm{eV}$ and . . 31. $3 \mathrm{eV}$

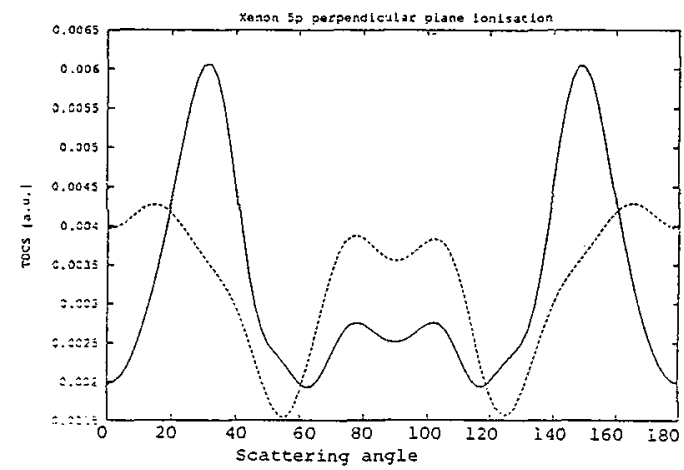

Figure $8 \mathrm{Xe}(5 \mathrm{p})$ perpendicular plane ionisation at $94.6 \mathrm{eV}$ incident electron energy; PVDTVBA approximation to triple differential cross section. DWBA and $\ldots \ldots$ 
[2] Ehrhardt H. and Rösel T.(1993) in "(e,2e) and related processes"

C.T.Whelan, H.R.J.Walters, A.Lahmam-Bennani and H.Ehrhardt edited(Kluwer Academic Publishers: 1993)

[3] Jones S., Madison D.H. and Srivastava M.K. J.Phys. B,(1992) 25,1899

[4]Pan C. and Starace A. F. Phys. Rev. A 45,(1992), 4588-4603.

[5]Rösel T., Dupre C., Roder J., Duguet A., Jung K., Lahmam-Bennani A. and Ehrhardt H. J. Phys. B 24 (1991) 3059-67

[6]Murray A.J. in "(e,2e) and related processes"

C.T.Whelan, H.R.J.Walters, A.Lahmam-Bennani and H.Ehrhardt edited(Kluwer Academic Publishers: 1993)

[7]Zhang X, Whelan C.T., Walters H.R.J., J.Phys.B,23 ,(1990),L173

[8] Clementi E. and Roetti C. Atomic Data and Nuclear Data Tables 14 (1974)

[9] Furness J.B. and McCarthy I.E. J. Phys. B 6 (1973) 2280-91

[10]Riley M.E. and Truhlar D.G. J. Chem Phys.63 (1975) 2182

[11]Pochat A., Tweed R.J., Whelan C.T., Zhang X., Walters H.R.J., Allan R.J., Gélébart F. and Cherid M. in "(e,2e) and related processes"

C.T.Whelan, H.R.J.Walters, A.Lahmam-Bennani and H.Ehrhardt edited(Kluwer Academic Publishers: 1993)

[12] Botero J. and Macek J.H. Phys. Rev. Lett. 68 (1992) 576 